\title{
Lithium-rotation connection in the newly discovered young stellar stream Psc-Eri (Meingast 1) (Corrigendum)
}

\author{
J. Arancibia-Silva ${ }^{1,2}$, J. Bouvier ${ }^{3}$, A. Bayo ${ }^{1,2}$, P. A. B. Galli ${ }^{4}$, W. Brandner ${ }^{5}, \mathrm{H}$ Bouy ${ }^{4}$, and D. Barrado ${ }^{6}$ \\ 1 Instituto de Física y Astronomía, Universidad de Valparaíso, Valparaíso, Chile \\ e-mail: javier.arancibia@postgrado.uv.cl \\ 2 Núcleo Milenio Formación Planetaria - NPF, Universidad de Valparaíso, Av. Gran Bretaña 1111, Valparaíso, Chile \\ 3 IPAG, Univ. Grenoble Alpes, 38000 Grenoble, France \\ ${ }^{4}$ Laboratoire d'Astrophysique de Bordeaux, Univ. Bordeaux, CNRS, B18N, Allée Geoffroy Saint-Hilaire, 33615 Pessac, France \\ 5 Max Planck Institute for Astronomy, Heidelberg, Germany \\ 6 Depto. Astrofísica, Centro de Astrobiología (INTA-CSIC), ESAC Campus, Camino Bajo del Castillo s/n, 28692 Villanueva de la \\ Cañada, Spain
}

A\&A 635, L3 (2020) https://doi .org/10.1051/0004-6361/201937137

Key words. stars: low-mass - stars: pre-main sequence - stars: abundances - open clusters and associations: individual: Psc-Eri stars: rotation - errata, addenda

In the original paper (Arancibia-Silva et al. 2020), there was a typographic error in Table 1 , where column $G_{b p}-G_{r p}$ was duplicated from column $M_{G}$ and the actual values referring to the quantity were not displayed. However, this has no impact on the analysis, results, or discussion in the paper since the correct values were used in the analysis (see, for example, Fig. 1 of Arancibia-Silva et al. 2020). The complete table with the correct values is presented in this erratum in Table 1.

\section{References}

Arancibia-Silva, J., Bouvier, J., Bayo, A., et al. 2020, A\&A, 635, L13 Curtis, J. L., Agüeros, M. A., Mamajek, E. E., Wright, J. T., \& Cummings, J. D. 2019, AJ, 158, 77 
Table 1. Stellar sample properties from Curtis et al. (2019) and EW(Li), radial velocity $\left(\mathrm{V}_{r}\right)$, and projected rotational velocity $(v$ sin $i)$ measurements

\begin{tabular}{|c|c|c|c|c|c|c|c|c|c|c|c|c|c|}
\hline Gaia DR2 & Curt & $\begin{array}{l}\text { RA(2000) } \\
\text { hh:mm:ss }\end{array}$ & $\begin{array}{c}\text { DEC(2000) } \\
\text { dd:mm:ss }\end{array}$ & $\begin{array}{c}T_{\text {eff }} \\
\mathrm{K} \\
\end{array}$ & $\begin{array}{c}\mathrm{G} \\
\mathrm{mag}\end{array}$ & $\begin{array}{c}M_{G} \\
\mathrm{mag}\end{array}$ & $\begin{array}{c}G_{b p}-G_{r p} \\
\text { mag }\end{array}$ & $\begin{array}{c}P_{\text {rot }} \\
\mathrm{d}\end{array}$ & $\begin{array}{c}\mathrm{EW}(\mathrm{FeI}) \\
\mathrm{m \AA}\end{array}$ & $\begin{array}{c}\mathrm{EW}(\mathrm{Li}) \\
\mathrm{m \AA}\end{array}$ & $\begin{array}{c}\mathrm{rms} \\
\mathrm{m} \AA\end{array}$ & $\begin{array}{c}\mathrm{V}_{r} \\
\mathrm{~km} \mathrm{~s}^{-1} \\
\end{array}$ & $\begin{array}{c}v \sin i \\
\mathrm{~km} \mathrm{~s}^{-1}\end{array}$ \\
\hline 2349094158814399104 & $79^{(*)}$ & $00: 47: 18.0$ & $-22: 45: 08.1$ & 4585 & 10.929 & 6.051 & 1.366 & 1.3 & 17 & 202 & 10 & 29.36 & 55 \\
\hline 4975223840046231424 & 81 & $00: 47: 38.5$ & $-47: 41: 45.8$ & 4551 & 11.514 & 6.985 & 1.42 & 5.8 & & $<50$ & & 15.67 & 7 \\
\hline 2355466790769878400 & 96 & $00: 55: 21.5$ & $-21: 24: 03.7$ & 4135 & 12.341 & 7.578 & 1.689 & 6.84 & & $<30$ & & 17.63 & $<6$ \\
\hline 5029398079322118912 & $52^{(*)}$ & $01: 13: 42.4$ & $-31: 11: 39.6$ & 5003 & 10.804 & 5.959 & 1.088 & 6.64 & 14 & 196 & 5 & 20.24 & $<6$ \\
\hline 4984094970441940864 & 101 & 01:21:49.7 & $-42: 01: 22.3$ & 4113 & 12.731 & 8.099 & 1.852 & 5.45 & & $<30$ & & 20.61 & 7 \\
\hline 2484875735945832704 & 65 & $01: 24: 24.7$ & $-03: 16: 39.0$ & 4842 & 11.791 & 6.398 & 1.222 & 8.4 & 15 & 82 & 13 & 16.84 & 7 \\
\hline 2491594263092190464 & 56 & 02:10:22.3 & $-03: 50: 56.7$ & 4944 & 11.533 & 6.153 & 1.136 & 2.26 & 14 & 225 & 10 & 19.37 & 19 \\
\hline 5117016378528360448 & 91 & $02: 17: 14.6$ & $-27: 16: 41.9$ & 4308 & 12.551 & 7.451 & 1.591 & 5.92 & & $<30$ & & 21.65 & 8 \\
\hline 5118895478259982336 & $90^{(*)}$ & 02:26:07.0 & $-24: 54: 49.0$ & 4431 & 11.728 & 6.658 & 1.579 & 5.61 & 18 & 31 & 5 & 21.83 & 10 \\
\hline 2488721720245150336 & 87 & $02: 26: 53.3$ & $-05: 17: 45.2$ & 4444 & 12.485 & 7.198 & 1.498 & 5.2 & & $<30$ & & 21.19 & 8 \\
\hline 5129876953722430208 & 76 & $02: 29: 28.5$ & $-20: 12: 16.8$ & 4829 & 11.695 & 6.739 & 1.334 & 8.0 & 15 & 99 & 5 & 22.7 & 7 \\
\hline 2496200774431287424 & $36^{(*)}$ & $02: 30: 58.8$ & $-03: 03: 04.9$ & 5448 & 10.415 & 5.328 & 0.928 & 5.45 & 11 & 173 & 5 & 22.25 & $<6$ \\
\hline 5179037454333642240 & 64 & 02:39:10.9 & $-05: 32: 22.5$ & 4859 & 11.765 & 6.373 & 1.215 & 6.42 & 15 & 125 & 5 & 19.23 & 8 \\
\hline 5161117923061794688 & 74 & 02:59:52.0 & $-09: 47: 35.8$ & 4800 & 12.063 & 6.639 & 1.308 & 5.45 & 15 & 184 & 10 & 20.44 & 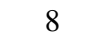 \\
\hline 5045955865443216640 & 40 & 03:00:46.9 & $-37: 08: 01.5$ & 5434 & 10.323 & 5.363 & 0.976 & 3.9 & 11 & 198 & 10 & 21.91 & $<6$ \\
\hline 5159567164990031360 & $88^{(*)}$ & 03:04:46.0 & $-12: 16: 57.9$ & 4330 & 12.613 & 7.098 & 1.56 & 0.45 & 19 & 50 & 30 & 19.27 & 55 \\
\hline 7324465427953664 & 61 & 03:05:14.1 & $+06: 08: 53.5$ & 4947 & 12.043 & 6.247 & 1.197 & 4.4 & 14 & 195 & 10 & 18.61 & 10 \\
\hline 5103353606523787008 & $46^{(*)}$ & 03:18:03.8 & $-19: 44: 14.2$ & 5077 & 10.473 & 5.227 & 1.058 & 1.26 & 13 & 242 & 5 & 20.41 & 25 \\
\hline 5106733402188456320 & $71^{(*)}$ & $03: 24: 25.2$ & $-15: 50: 05.4$ & 4944 & 11.517 & 6.197 & 1.278 & 0.62 & 14 & 245 & 10 & 40.06 & 61 \\
\hline 5168681021169216896 & 29 & $03: 29: 30.3$ & $-07: 10: 13.8$ & 5478 & 10.827 & 5.204 & 0.895 & 5.32 & 11 & 168 & 5 & 20.78 & 8 \\
\hline 3247412647814482816 & 80 & $03: 32: 30.9$ & $-06: 13: 09.1$ & 4714 & 12.327 & 6.898 & 1.382 & 6.66 & 16 & 34 & 10 & 22.87 & 8 \\
\hline 5114686272872474880 & 69 & $03: 47: 25.8$ & $-12: 32: 30.9$ & 5006 & 12.634 & 6.55 & 1.247 & 9.0 & 14 & 61 & 15 & 20.15 & 7 \\
\hline 4842810376267950464 & 38 & $03: 47: 56.3$ & $-41: 56: 24.9$ & 5355 & 10.762 & 5.383 & 0.936 & 5.7 & 12 & 143 & 5 & 18.75 & $<6$ \\
\hline 3243665031151732864 & 50 & $03: 48: 38.3$ & $-06: 41: 52.6$ & 5063 & 11.46 & 5.953 & 1.082 & 6.84 & 13 & 151 & 5 & 22.04 & $<6$ \\
\hline 3245140743257978496 & $43^{(*)}$ & 03:54:01.0 & $-06: 14: 14.6$ & 5344 & 11.146 & 5.434 & 1.017 & 5.66 & 12 & 193 & 15 & 21.7 & 8 \\
\hline 3193528950192619648 & 41 & 03:57:04.0 & $-10: 14: 00.9$ & 5270 & 11.297 & 5.534 & 0.994 & 5.54 & 12 & 195 & 5 & 20.88 & $<6$ \\
\hline 5083255496041631616 & 49 & $03: 57: 35.1$ & $-24: 28: 42.2$ & 5078 & 11.131 & 5.885 & 1.077 & 6.08 & 13 & 7 & 5 & 22.98 & $<6$ \\
\hline 5097262136011410944 & 62 & 03:58:54.7 & $-17: 05: 53.2$ & 4899 & 11.649 & 6.311 & 1.199 & 7.58 & 14 & 105 & 5 & 22.45 & 7 \\
\hline 5096891158212909312 & 59 & $04: 12: 46.0$ & $-16: 19: 29.1$ & 4907 & 11.994 & 6.187 & 1.181 & 3.68 & 14 & 255 & 10 & 21.25 & 13 \\
\hline 4871041608622321664 & $60^{(*)}$ & $04: 28: 28.9$ & $-33: 53: 45.1$ & 4924 & 11.63 & 6.037 & 1.193 & 6.5 & 14 & 215 & 10 & 21.21 & 7 \\
\hline 2594993646533642496 & 67 & $22: 31: 13.9$ & $-17: 04: 52.4$ & 4829 & 11.934 & 6.451 & 1.242 & 6.1 & 15 & 174 & 10 & 9.96 & $<6$ \\
\hline 2402197409339616768 & $57^{(*)}$ & 22:39:01.4 & $-18: 52: 55.7$ & 4921 & 11.219 & 6.155 & 1.142 & 7.8 & 14 & 115 & 5 & 13.09 & $<6$ \\
\hline 2596395760081700608 & 53 & $22: 39: 53.5$ & $-16: 36: 23.3$ & 5065 & 11.508 & 5.994 & 1.097 & 6.97 & 13 & 126 & 5 & 10.75 & 7 \\
\hline 2433715455609798784 & 70 & $23: 36: 52.1$ & $-11: 25: 01.7$ & 4810 & 11.737 & 6.442 & 1.249 & 6.3 & 15 & 214 & 10 & 14.66 & 8 \\
\hline 2393862836322877952 & $66^{(*)}$ & $23: 40: 37.5$ & $-18: 11: 37.9$ & 4734 & 11.485 & 6.472 & 1.239 & 7.7 & 16 & 99 & 10 & 16.88 & 7 \\
\hline 2390974419276875776 & 72 & $23: 48: 32.4$ & $-18: 32: 57.4$ & 4706 & 11.583 & 6.618 & 1.283 & 6.5 & 16 & 123 & 10 & 17.14 & 8 \\
\hline 2418664520110763520 & 63 & $23: 49: 55.1$ & $-15: 43: 42.0$ & 4917 & 11.607 & 6.396 & 1.213 & 2.94 & 14 & 215 & 10 & 16.87 & 15 \\
\hline 2339984636258635136 & 77 & $23: 56: 53.7$ & $-23: 17: 24.6$ & 4545 & 11.475 & 6.765 & 1.349 & 8.35 & 17 & 22 & 5 & 15.8 & 7 \\
\hline
\end{tabular}

Notes. ${ }^{(*)}$ Bona fide binary systems. 